\title{
Vaccines for the Third World
}

Barry R. Bloom

New vaccines, developed through genetic engineering, can make immunization an even more effective weapon for tackling disease in developing countries. So what is preventing progress?

Where there is no vision, the people perish ... Proverbs 29:18

THE Third World is the place where threequarters of the people of this planet live, where 86 per cent of all births and 96 per cent of child and infant deaths occur ${ }^{1,2}$. At both a national and a human level the diverse problems of the developing countries are of staggering proportions. Most have heavy foreign debt and, as a consequence, these countries now transfer to the developed countries more hard currency than they receive $(\$ 31.1$ billion in 1987$)^{3}$. Most are burdened by disease (Table 1 , see over) ${ }^{4}$; the reality is that millions of people are sick because they are poor, and poorer because they are sick. Some indicators of the quality of life of people of the 40 poorest countries are listed in Table 2 (see over), and the trends have not been encouraging. Per capita income has declined over the past five years and the percentage of national budgets spent on health has been unchanged or has diminished for eight years.

Yet one aspect of life there has improved profoundly. The number of children receiving immunizations has risen from 5 per cent in 1974 to over 60 per cent this year. The World Health Organization Expanded Programme for Immunization (EPI) prevented the deaths of 2.2 million children last year. Through the efforts of 25,000 professional national and international staff and hundreds of thousands of field workers, 60 million children are now vaccinated annually against diphtheria, pertussis, tetanus (DPT), polio, measles and tuberculosis. The number of cases of paralytic poliomyelitis has declined in the Americas from 4,500 ten years ago to fewer than 200 this year, and WHO has just made the eradication of polio one of its goals ${ }^{5}$. Immunization is the most cost-effective weapon for disease prevention in developing countries, and new molecular and genetic technologies are making new types of vaccines feasible. The eradication of smallpox demonstrated that they can be effective everywhere. What is lacking is the will to make the advances of modern biomedical science available to the poorest people of the world.

Vaccine problems and constraints. As impressive as the results of global childhood immunization have been, there are many diseases for which vaccines are not available, as well as inherent limitations in each of the currently used vaccines. Only two EPI vaccines - BCG, used to immunize against tuberculosis, and oral polio vaccine - can be given at birth or any time thereafter, and BCG and measles are the only vaccines that require a single immunizing dose. DPT and polio must be given

\section{IMAGE UNAVAILABLE FOR COPYRIGHT REASONS}

This was smallpox, a disease that has been eradicated through immunization.

several times to achieve protective levels of antibodics. Bccause matcrnal antibodies circulating in the infant inactivate the vaccines, DPT can be given only at six weeks of age, and then at monthly intervals up to 14 weeks. The dropout rate is high - about 20 per cent fail to return for each required booster shot. In the case of the vaccine against measles, which is responsible for the death of 2 million children annually in the developing world, the presence of maternal antibodies that neutralize the vaccine in young children represents a 'wall' that scientists have not yet been able to hurdle. If measles vaccine were given to children in developing countries at $12-15$ months, the time recommended in the developed world, up to $30-50$ per cent would have contracted the disease before receiving the vaccine. Measles vaccine is given at $9-12$ months and a new strain may even be effective at 6 months. In all, a total of five contacts are required between the child and the health services, which represents a considerable logistical problem.
Any new or improved vaccine to be considered for inclusion in the EPI should, ideally, have the following attributes: (1) it must be inexpensive; (2) it must be safe; (3) it must be extremely effective, inducing protection in $90-100$ per cent of recipients; (4) it should engender lifetime immunity; (5) it should be heat-stable and not need to be kept cold at all stages (a cold chain), which is both expensive and subject to catastrophe; (6) it should require only one shot or be compatible with the schedule for other vaccines; (7) it should be simple to give, and because of the problems of reuse of needles and of HIV infection it should be given by a non-invasive route; and (8) it should be capable of being given as close to birth as possible. Dr W. Foege, Chairman of the Task Force on Child Survival, has described the ideal vaccine as one in which "single administration at birth will provide protection from multiple diseases".

New vaccines from old. There are basically four kinds of vaccine, each of which has strengths and limitations for use in the Third World.

- Killed vaccines are among the simplest and least expensive to prepare. They contain many antigens and assure some responsiveness in virtually all individuals. But even under defined production conditions, some vary in reproducibility, and all require careful monitoring to assure that no viable organisms are present. As in the case of pertussis, killed vaccines often have a higher level of reactigenicity or toxicity than is desirable.

- Subunit vaccines prepared from individual components of a pathogen, by chemical synthesis or recombinant DNA technology (for example tetanus and diphtheria toxoids) have several advantages. They are chemically defined, reproducibly prepared and assayed, and are usually inexpensive to manufacture. Carbohydrate antigens are important for protection against many pathogens. Because complex carbohydrates cannot be produced by recombinant DNA technology, subunit vaccines, particularly carbohydrate-carrier-protein conjugate vaccines, are the only feasible strategy at present for producing protective immunity to pathogens such as Hemophilus influenza b and Streptococcus pneumoniae, which cause meningitis, mental retardation and pneumonia. 
A general drawback of both killed and subunit vaccines is the need for several immunizations and boosters - immunological memory benefits from repeated stimulation by antigens. Some chemically defined subunit or peptide vaccines contain a desired antigenic determinant (epitope) that elicits B-cell-produced neutralizing antibodies, but they may not engender immunological memory to the infectious agent which requires expansion of specific T-helper cells as well; thus reinfection will not result in a boosting of the immune response. The possible use of small synthetic epitopes in subunit vaccines raises the concern that polyclonal antibodies developed against a single epitope of a pathogen may function very much like monoclonal antibodies and select for escape mutants - that is, antigenic mutants with altered epitopes on neutralizing antigens that are not neutralized by existing antibodies in the population. Although this would not be catastrophic for a single epitope in the first instance, new antigenic mutants could be created that would, in time, accumulate and possibily become resistant to normal acquired immune defences.

- Live attenuated vaccines have largely been derived by empirical methods, and those in current use have the advantages of generally inexpensive production, persisting immunity and a good safety record. Nevertheless, there are considerable problems with existing live vaccines in terms of reversion to virulence (polio, Sabin type 3 ), reactigenicity (BCG), need for a cold chain, and possible induction of disease in immunodeficient and some normal individuals (for example polio).

- Anti-idiotype vaccines are a novel concept that has yet to be realized. They are based on the principle that because antibody active sites (the idiotype) are complementary to the specific antigenic determinant to which they bind, some antibodies raised against a particular antibody active site may indeed mimic antigen. Although immunization has been achieved in some experimental systems with anti-idiotype or anti-antibody vaccines, so far there has been difficulty in achieving high levels of immunization and immunological memory because $T$ cells are not developed against antigens of the pathogen. It remains to be seen in what circumstances anti-idiotype vaccines will prove to be useful.

In considering the various criteria for new vaccines for the Third World, genetically engineering live attenuated vaccines to become multivaccine vectors that can immunize simultaneously against multiple antigens is particularly appealing. Two basic strategies are being developed, one using viral vaccines, the other using bacterial vaccines as recombinant multivaccine vehicles. Viral vaccines in general
TABLE 1 Diseases of the Third World that potentially could be prevented by vaccines*

\begin{tabular}{lcc}
\hline Condition & $\begin{array}{l}\text { Deaths per } \\
\text { year (million) }\end{array}$ & $\begin{array}{c}\text { Estimated } \\
\text { episodes } \\
\text { or incidence } \\
\text { year (million) }\end{array}$ \\
Respiratory & 10 & 15 \\
$\quad$ disease & 4.3 & 28 \\
Diarrhoea & $3^{\dagger}$ & 10 \\
Tuberculosis & 2 & 67 \\
Measles & 1.5 & 150 \\
Malaria & 0.8 & 3.7 \\
Hepatitis B & 0.35 & 1 \\
Meningitis & 0.33 & 10 \\
Schistosomiasis & $0.1^{\ddagger}$ & 0.75 \\
AlDS & $<0.06$ & 4,900 \\
Worm infections & & \\
\hline
\end{tabular}

* Modified from J.A. Walsh (ref. 4).

† Data from WHO Tuberculosis Unit.

$\mp$ Data from WHO, unpublished estimates.

TABLE 2 Indicators of the quality of life in the world's 40 poorest countries, 1988

Average annual gross national product per capita

$\$ 270$

Literacy

Males

Females

Population with access to water Urban

Rural

$61 \%$

Government expenditure

Health

Education

Defence

$5.5 \%$

$14 \%$

Population per physician with access to health services

Life expectancy at birth

$48 \mathrm{yr}$

Infant mortality (<1yr per 1,000 births)

Mortality rate of children aged under 5 (per 1,000 births)

Population annual growth rate (1973-84)

Children suffering mainutrition

Children immunized with DPT, polio, measles and BCG

have certain advantages: they express antigens in eukaryotic cells with correct folding, proteolytic processing, glycosylation, secretion and subunit assembly; and they can stimulate the production of cytotoxic T-lymphocytes as well as antibodies. Bacterial vaccines have a special ability to immunize for long periods and engender local immunity, for example in the gut, and cell-mediated immunity.

The technology: the power of molecular blology and genetics. The ability to change living organisms by genetic engineering has given rise to the possibility of devising vaccine vehicles that can immunize simultaneously against antigens of different pathogens. Such multivaccine vehicles can be developed from either viral or bacterial vaccines, and each approach has its particular advantages.

- Live attenuated viral vaccine vehicles. Smallpox was eradicated by the use of live attenuated strains of vaccinia virus, originally derived by Jenner from a cowpox virus. Although vaccinia may be of somewhat dubious provenance, it has been astoundingly effective. The virus is a large double-stranded DNA virus of about 185,000 base pairs that contains all the information for its own transcription and replication in the cytoplasm of a wide range of host cells. Large amounts of DNA, approximately 25,000 base pairs, are expendable and not required for vaccinia replication. The molecular strategy to develop vaccinia into a multivaccine vehicle is based on the ability to replace non-essential viral DNA with foreign genes, and have them expressed under the control of the vaccinia-virus promoters and transcription system. Plasmids have been constructed containing one or more promoters, cloning sites for introducing DNA for foreign antigen genes, and a selectable marker, all flanked by DNA sequences that complement segments of non-essential DNA of the virus. When cells containing the plasmid are infected with vaccinia virus, a low frequency of double recombinations can occur between the flanking DNA sequences surrounding the foreign DNA in the plasmid and the non-essential DNA of the virus, resulting in the precise replacement of the viral DNA sequences by the desired promotor and foreign antigen gene. A wide range of viral, bacterial and protozoal antigens has been expressed in vaccinia, as many as four antigens being expressed simultaneously in a single recombinant virus ${ }^{6}$.

The principal disadvantage of vaccinia is the frequency of complications, which approaches the limits of current acceptability. Although only 1 in 300,000 recipients of the least troublesome vaccinia strain developed severe neurological effects, the incidence of serious complications, including disseminated vaccinia and vaccinia gangrenosum, was of the order of 1 in 1,000 in New York State, and is certainly higher in some developing countries ${ }^{7}$. (It is regrettable that data on complications in developing countries are not available from the global eradication campaign.) By genetic engineering it should be possible to define viral genes involved in neurovirulence and reactigenicity and to create new, less reactigenic, vaccine strains. It has also been possible to incorporate genes for lymphokines such as interleukin-2 to enhance immune responses to foreign antigens and the virus itself.

Because recipients produce neutralizing antibodies to the virus, a second 
limitation of vaccinia is that it is not feasible, at least in the short term, to give booster shots, because reintroduced recombinant vaccinia will simply be neutralized. Practically speaking, vaccinia is a one-shot vaccine by necessity, and that shot must be highly effective to make it useful. Recombinant vaccinia expressing the glycoprotein antigen of rabies virus is extraordinarily immunogenic and, despite some uncertainty about vaccinia's acceptability in human beings, recombinant vaccinia has recently been introduced in Belgium, and a region of France, in bait to protect foxes against rabies. More importantly, perhaps, there is an epidemic in West Africa of rinderpest, a cattle disease caused by a measles-like morbillavirus, and the International Commission of Epizootics plans to immunize hundreds of millions of cattle with recombinant vaccinia expressing rinderpest antigens. In an experiment of that scale, with perhaps 100,000 vaccinators, there is bound to be adventitious infection of human beings with the recombinant vaccine. It will be essential that they are pre-immunized against vaccinia virus.

Adenovirus vaccines have been used in tens of millions of US military recruits to protect against respiratory disease, essentially without serious complications. Curiously, the vaccine is not an attenuated strain, but rather consists of two virulent strains, types 4 and 7 , that are given by an unnatural route, orally, that immunizes without producing disease. The use of enteric-coated lyophilized vaccine tablets represents a distinct advantage for delivery. The virus has a rather small DNA genome, but a limited number of foreign genes have been introduced and expressed in adenoviruses by a similar, precise gene-replacement strategy, including the hepatitis-B surface antigen that is not well expressed in vaccinia ${ }^{8}$. Concerns include the role of the $E 1 A$ gene, which functions like certain protooncogenes in transformation of cells in culture, and the fact that the safety of existing adenovirus vaccines in children is unknown. Herpes viruses are large DNA viruses that, like vaccinia, have large amounts of expendable DNA; they could be developed into recombinant multivaccine vehicles, provided that the genes for neurovirulence can be identified and deleted.

Two RNA viruses offer promise as recombinant vaccine vectors. The live attentuated polio vaccine is one of the most effective vaccines in the developed world, but it has been disappointingly ineffective in many developing countries with up to 30 per cent of recipients failing to produce acceptable neutralizing titres, generally to type 3 . There has been a return to multiple injections of killed polio vaccine in some places. Polio vaccines are not without problems, even in the developed countries, because essentially all cases of poliomyelitis there result from reversion of vaccine strains to virulence. Because Sabin type 3 vaccine differs from virulent poliovirus type 3 by only two mutations, reversion to virulence is an intrinsic problem.

The key to the genetic manipulation of polio was the discovery that artificially produced complementary DNA to the viral RNA is infectious and, on transfection of cells, produces infectious poliovirus. Of the several neutralizing epitopes on polioviruses, all but one are conformational and composed of discontinuous sequences of amino acids, the exception being a single loop of linear sequence? Several laboratories have been able to recombine cDNA sequences of the loop that generate the main neutralizing epitopes, for example, from type 3 into the very stable polio type 1 vaccine, to create hybrid vaccines that generate neutralizing antibodies to both types 1 and 3 poliovirus $^{10-12}$. The cDNA sequences encoding the loop can be modified to express sequences for small epitopes of foreign antigens, including HIV. At present, however, there is no evidence that these recombinant vaccines can immunize by oral administration.

One of the safest and most effective live attenuated viral vaccines known is the $17 \mathrm{D}$ vaccine against yellow fever. A single immunization results in sustained neutralizing titres for periods of over 40 years, and complications are very rare. It has recently been possible to produce infectious cDNA from the $17 \mathrm{D}$ vaccine ${ }^{13}$ and one hopes that the possibility of developing $17 \mathrm{D}$ as a recombinant vaccine vector will be rapidly explored.

- Live attenuated bacterial vaccine vehicles. The first modern approach to a rationally designed live attenuated vaccine was the production, by chemical mutagenesis, of an auxotrophic mutant of the virulent bacterium Salmonella typhi. A mutant in galactose epimerase (GalE) was selected that could not convert UDPgalactose to UDP-glucose. This strain, Ty21a, retained the ability to infect after oral administration and to colonize the gut, essential for producing secretory immunity. The mutation was designed to be a time-bomb; Ty21a can grow for several days, and then, when it accumulates more UDP-galactose than it can tolerate, it dies, liberating antigens. This strain has proven to be remarkably stable and safe, and has been tested in field trials in Egypt and Chile against typhoid fever.

Ty21a suffers from two disadvantages. One never knows precisely what gene(s) is affected by chemical mutagenesis. Indeed, when the GalE gene from $S$. typhi was deleted by another group and the deleted strain was tested in volunteers, two out of the four recipients developed typhoid fever, revealing that the key

\section{IMAGE UNAVAILABLE FOR COPYRIGHT REASONS}

The campaign against smallpox continued even after the last endemic case. This poster advertises a $\$ 1,000$ reward for anyone reporting an occurrence.

attenuating mutation in Ty21a must have been in a gene other than GalE. Equally distressing, three oral immunizations of Ty21a were required to achieve 67 per cent protection, an efficiency insufficient for a useful anti-typhoid vaccine or multivaccine vehicle.

To avoid the vagaries of chemical mutagenesis, the genetic strategy for salmonella vectors is to delete genes required for pathogenesis or survival, then to insert foreign genes into the chromosome by gene replacement, or express them on plasmids in the cytoplasm. A promising set of targets for gene deletion has been genes $(\operatorname{aro} A, C$ and $D)$ of the aromaticamino-acid pathway required for synthesis of folate and enterochelins (ironbinding proteins) as well as proteins ${ }^{14}$. These are auxotrophic mutants that cannot grow without added aromatic amino acids, and thus they too function as timebombs. Vast amounts of foreign genetic material can be introduced and expressed in bacteria, and these salmonella auxotrophic mutants have immunized animals against introduced recombinant antigens.

A second genetic strategy used to produce recombinant Salmonella typhimurium vectors has been deletion of the genes for adenylyl cyclase (cya) and the cAMP-binding protein ( $c r p$ - catabolite repressor protein) which, together with removal of a plasmid containing virulence genes, has eliminated pathogenicity. Genes for foreign antigens expressed on plasmids introduced into these strains have persisted and immunized mice ${ }^{15}$. The great advantage of salmonella recombinant vectors is that they can be given orally, and, in experimental animals, they immunize both locally and, at the T-cell level, systemically. 
Unfortunately, the few trials in human volunteers with recombinant salmonella vectors have indicated that the strains tested have been overattenuated and do not produce adequate secretory $\operatorname{IgA}$ antibodies. Patient efforts are required to construct salmonella strains that can achieve the delicate balance between appropriate attenuation and effective colonization leading to immunization. They must immunize not only against typhoid but against introduced recombinant antigens; of particular interest are those for cholera, shigella and enterotoxigenic E. coli.

The oldest and most widely used live attenuated vaccine in the world is, perhaps surprisingly, BCG - Bacille Calmette-Guerin - an attenuated bovine tubercle bacillus used to immunize against tuberculosis. This mycobacterial vaccine has been given to over 1.5 billion people with a low frequency of serious complications, although it has the highest rate of minor reactions of any of the EPI vaccines. Its effectiveness in protecting against pulmonary tuberculosis has varied greatly in different parts of the world, but BCG requires only a single shot to engender cell-mediated immunity for periods of $5-50$ years. It is known to be an effective adjuvant, enhancing immune responses to many different antigens. BCG can be administered at birth or at any time thereafter, and can be given repeatedly. Like most bacterial vaccines, it is very inexpensive ( $\$ 0.055$ per dose).

In contrast to $E$. coli, $\mathrm{BCG}$ grows very slowly and requires about 24 days to produce a colony ( $E$. coli takes 8 hours). Because it seemed unlikely that direct genetic manipulation of mycobacteria would prove efficient, a 'shuttle' strategy has been devised to introduce and express foreign genes in BCG. Basically, phages or plasmids were constructed that can replicate both in $E$. coli and in mycobacteria $^{16}$. Foreign genes and selectable markers can be introduced by standard molecular genetic techniques in $E$. coli, and the recombinant DNA is then 'shuttled' into BCG. Foreign genes can then be expressed either by replacing non-essential BCG chromosomal genes or from multicopy plasmids in the cytoplasm. It remains to be seen, however, how effective antigens known to be immunogenic when mixed with mycobacterial adjuvants are when expressed by the organism itself.

The spectre of AIDS. There is a serious risk with any live vaccine, namely dissemination and serious complications in immunodeficient individuals. The current HIV epidemic in many countries heightens concern about side effects from childhood vaccination. For example, vaccinia has been adminstered to US military personnel, 28 of whom were later found to be

The logistical problem - an immunization team in the Philippines sets out by canoe.

HIV seropositive. One died of generalized vaccinia. (One cannot help but wonder, as, by convention, study or use of smallpox (variola) virus has been discontinued and all strains presumably locked away in three safe repositories, why anyone in the world is being vaccinated against smallpox.) Several major cohort studies are underway in Africa to evaluate the consequences of standard childhood vaccination in HIV-seropositive compared to HIV-seronegative children. It is encouraging that so far no discernible differences in untoward complications of any of the vaccines have been observed. It may be that there is a window in time in congenitally infected children during which they can be immunized and before which any significant immunodepression occurs. Because children who will be immunocompromised are even more susceptible to the principal childhood diseases, particularly measles, it is strongly recommended by WHO that these children receive all the childhood vaccines, except for BCG in children with symptomatic immunodeficiency.

The basic premise of existing vaccines has long been the utilitarian principle of providing the greatest good for the greatest number of people; it has long been recognized that small numbers of individuals will suffer severe complications. At the very least, antidotes to restrict disseminated infection of recombinant live vaccines should be available (for example, vaccinia immune globulin, isoniazid for BCG, antibiotics for salmonella). Because of the high cost of drugs potentially useful against AIDS, it is sadly likely that most will not be available to patients in developing countries in the foreseeable future; consideration of cost effectiveness alone should spur efforts to develop effective vaccines against AIDS.

Single-dose, controlled-release vaccines. The requirement for several injections is the main limitation to subunit vaccines. Almost 800,000 neonates per year still die of tetanus, which can be prevented simply by immunizing women of childbearing age. Controlled-release systems are already being used in man (and cattle) to deliver an array of drugs and hormones. Some of these systems have been used by the WHO Special Programme on Human Reproduction (HRP), which has supported research on anti-fertility vaccines. Taking advantage of HRP expertise in the area, development of single-dose vaccines has become a goal of the WHO Special Programme for Vaccine Development with neonatal tetanus as its first target.

Basically, polymers of lactic and glycolic acids (PLA/PGA) approved for human use are being incorporated into controlled-release microspheres or microcapsules which can provide either continuous release of tetanus toxoid over a period of several months, or a pulsed release similar to booster shots, that would occur, for example, 4 and 8 weeks after a single immunization. Should this prove effective, DPT, and a number of other subunit antigens could be incorporated into this type of one-shot vaccine not the least important of which will be epitopes from human chorionic gonadotrophin or sperm to control fertility ${ }^{17}$.

Immunological problems. At a scientific level, the constraints on development of new vaccines are not likely to come from molecular biology and genetics; rather they will be biological and immunological. 
Although antigens have been cloned from virtually every known pathogen, and many $\mathrm{T}$ and $\mathrm{B}$ epitopes have been mapped on various antigens, there are very few pathogens for which the mechanism of protection is understood. Nor is it clear which antigens are required to engender those protective responses.

A multitude of unanswered questions remain. Must a protective vaccine against malaria sporozoites generate antibodies, or $\mathrm{T}$ cells, or both? Can production of specific immunoglobulin isotypes be targeted (for example IgA for intestinal pathogens, IgE for killing schistosomes, IgG2 for carbohydrate antigens)? Can the type of $\mathrm{T}$ cells (for example helper $\mathrm{T}$ cells, cytotoxic T-cells, gamma-delta $\mathrm{T}$ cells, and lymphokines) produced be controlled (for example by targeting the vaccine to the cytoplasmic compartment or endosomal compartments of antigenpresenting cells)? Can T-cell responses against important protective antigens be generated in individuals of all histocompatibility types? Can nonvarying epitopes be found on highly polymorphic protective antigens that can be effective against genetic variants of the pathogens (for example malaria, HIV, African trypanosomes)?

At the same time, immunology offers novel solutions for basic vaccine problems. Measles vaccine cannot be used in children under the age of 6-9 months because it is inactivated by maternal antibodies, but it is conceivable that appropriate measles $T$-cell epitopes could be given at birth in one of the recombinant vaccine vectors, such that T-cell memory is generated to measles and a protective response would follow natural infection. Clearly, the easy vaccines have already been made; new vaccines pose greater challenges for research.

'It is only a matter of implementation'. There are few more portentous words than these to be found in any health document. For most scientists engaged in the development of new health interventions, the fulfilment of their research is a product that goes through clinical testing and is eventually licensed for use. In international health, the historical record belies such optimism. For example, all of the vaccines in the EPI programme were available before 1974 , but only 5 per cent of the world's children received them - it was clearly only a matter of implementation.

Certainly, the main limiting factor is cost, but it is not the only one. The scientific infrastructure for evaluating new drugs and vaccines relevant to Third World diseases is also limiting. It is difficult and expensive to carry out clinical trials in industrialized countries in which the diseases do not occur. From the beginning of the WHO-Tropical Disease
Research (TDR) programme a component was mandated in addition to the scientific research programmes for "institutional research strengthening" in the countries worst afflicted. This support, representing 25 per cent of the budget, has been used to set up and modernize laboratories, and to train bright students abroad (and, occasionally, even to make it sufficiently attractive for them to return home). As a consequence, in many developing countries there are laboratories that are able to tackle tropical diseases.

What has been largely overlooked, however, is the role of field and epidemiological research in developing countries. Almost all incentives - financial, working conditions and personal recognitionmotivate people to go into medical practice, laboratory-based research or, most commonly, administration. There are few rewards for health workers in the field. Yet the field worker is the mainstay for acquiring information about local health problems, for evaluating new interventions and for integrating, maintaining and monitoring them in control programmes. Recognition of their importance through the development of appropriate career structures and educational and material incentives is a major challenge to the developing countries.

At the same time, the development of new drugs and vaccines ( 38 new products that have resulted from the WHO-TDR programme are currently in field trials) provides a unique opportunity for building scientific and field infrastructures in the Third World. Field-research and control infrastructures need no longer be tied directly to one drug or vaccine, but can be continuing mechanisms to assess different health strategies and disease-control activities. Much of the same technology enzyme-linked immunosorbent assays (ELISA) or polymerase chain reactions (PCR), for example - can be used in the same place to assess the distribution of parasites in mosquitos and the incidence of multiple infections by multiple pathogens in the population. The epidemiological tools for testing different drugs against one disease are often applicable to trials against another and can be adapted to evaluating vector control and vaccines as well. The result of this research is not papers; it is the control of disease.

A vital aspect of implementation has almost invariably been ignored. Fifteen years ago it became clear that although oral polio vaccine is superbly effective in developed countries, it was proving disappointing in developing countries. Studies show that only $40-80$ per cent of children receiving oral polio vaccine produce antibodies sufficient to ensure protection. To this day, it is unclear why oral polio vaccine is so ineffective in developing countries. Is it that children are already colonized with enteric viruses that have a competitive advantage over the vaccine, or that maternal antibodies neutralize the vaccine? Or is it simply that the vaccine sold in the United States contains six times more viral particles?

Until two years ago, EPI's mandate was 'implementation' only, and it lacked a research component. The tragedy, measured in lost lives, has been misapprehension that there can be implementation without research. In this context, I mean not only laboratory-based research, but social and economic research to provide information on costs and benefits, and to provide the means to understand how best to design and implement control programmes and identify and overcome obstacles to their effectiveness.

The main trend in development since the Second World War, as the Nobel prize-winning economist Theodore Schultz pointed out ${ }^{18}$, has been investment in physical capital - dams, factories and so on - with little recognition of the potential for investment in 'human capital'. The role of health, education and quality of life in development has generally been underestimated, because it is much easier to measure direct industrial and agricultural productivity than the indirect political and economic effects of health and knowledge on the economies or political stability of developing countries.

Vaccines are clearly not the key to development. Yet they can and do serve as an entry point both to strengthening science and primary health-care infrastructures. The infrastructure for vaccines can be extended to other low-cost/highimpact medical technologies on a mass scale, including oral rehydration, and maternal and child care and education, especially breastfeeding, food supplementation and family planning. In 1984, for example, Colombia vaccinated three-quarters of its children under five years of age through three national immunization days, and has subsequently mobilized complementary efforts for primary health care and education.

Much ink has been spilled on "appropriate technology' for the Third World, but there has been almost no consideration of 'appropriate science'. Research on vaccines involves a knowledge of molecular biology, genetics, immunology and epidemiology, and could serve as a stimulus to the educational and scientific advancement important to development. Even the poorest countries have need for expertise and access to biomedical science; they cannot afford to squander their resources on iron lungs.

The bottom line. It is ultimately, one supposes, a matter of priorities. The annual budget for the entire World Health Organization is $\$ 327$ million. The TDR Programme for six tropical diseases has a 


\section{IMAGE \\ UNAVAILABLE FOR COPYRIGHT REASONS}

Out of the laboratory and into practice - announcement of an immunization day in Bolivia.

budget of $\$ 30$ million, and the budget for the Special Programme for Vaccine Development has a budget of $\$ 2.8$ million. The annual cost of childhood immunization is some $\$ 500$ million, averaging approximately $\$ 10$ per immunized child. The vaccines themselves represent less than 10 per cent of the cost of immunization; of the remaining costs for personnel, transport and logistics, 70 per cent is borne by the countries themselves. The major advance in vaccine coverage has been accomplished largely through the combined efforts of the EPI of WHO, UNICEF, The World Bank, and the UN Development Programme, with support from non-governmental organizations, particularly the Rockefeller Foundation, Rotary International and the Save the Children Fund, which together coordinate their efforts through the Task Force for Child Survival. Behind the scenes scientists trying to develop new or improved vaccines scramble for support, most of it coming from the US National Institute for Allergy and Infectious Diseases or the Department of Defense, or from WHO's Special (extrabudgetary) Programmes for Research and Training in Tropical Diseases, Human Reproduction, or Vaccine Development. A decade ago, some foundations were generous contributors to research on diseases of the Third World, but their interest seems more to have reflected fashion than a serious commitment to the problem. And one must add that, regrettably, few Third World leaders have made health a high priority.

There has been only limited interest by the private sector in development of vaccines for the industrialized world, and virtually none in vaccines for the Third World $^{19}$. The reasons are simple. Vaccines account for only 1 per cent of the profits of the pharmaceutical industry, and a greater percentage of their liability. One of the two new vaccines licensed in the past ten years is a subunit vaccine against Hemophilus influenza $\mathrm{b}$, the main cause of meningitis and mental retardation in young children around the world. It consists simply of a bacterial carbohydrate conjugated to tetanus or diphtheria toxoids to augment the immunogenicity. The cost for each of the companies trying to develop that vaccine has been $\$ 8-12$ million. The cost would almost certainly be higher for live vaccine vectors.

Another example: hepatitis-B virus is not only the cause of a serious infectious disease, but the principal known risk factor for hepatocellular carcinoma, which is particularly prevalent in Asia. Several vaccines are currently being produced, one of which is available to EPI for largescale public health use at $\$ 1$ per dose. Nevertheless it cannot be included in EPI because of cost. Because of the numbers of people involved, there must be incentives to develop and deliver vaccines and essential drugs to the Third World. It should at least be possible to establish cost-plus agreements with international agencies to develop potentially useful interventions, or joint ventures or affiliations in developing countries.

The imagination and resources of the private sector that were so instrumental in developing biotechnology should be engaged, and the private sector may find that it has something both to contribute and to gain. Failing that, developing countries that can afford it will have to rely on their own abilities, intellectual and material, for developing their own biotechnology. This is already happening in Cuba, India, Brazil and Mexico. It is important to note that several new vaccines now in field trials have, in fact, been developed by scientists in the Third World - vaccines against leprosy (developed in Venezuela and India), leishmaniasis (Venezuela and Brazil) and dengue haemorrhagic fever (Thailand). Finally, Third World countries have enormous foreign debt, much of which will clearly have to be written off. If even a small portion of that debt were restructured to be spent in local currency for health and education, several of the problems affect- ing the quality of life in the Third World could be addressed.

The First World is the place where 13 per cent of the world's people consume the major part of the planet's crude resources, most of which come from the Third World. In the United States we spend annually over $\$ 300$ billion on 'defence'; a single B-2 'stealth' bomber costs $\$ 532$ million. In terms of personal consumption, $\$ 55$ billion ( $\$ 287$ per adult) is spent annually on alcohol, $\$ 38$ billion on tobacco and $\$ 22$ billion on toys ${ }^{20}$. We can afford to do more for health in the Third World. Conscience should motivate us to do so, and self-interest supports the claims of conscience. In the first place, good public health translates into good economics. We save over $\$ 0.5$ billion per year in no longer having to control smallpox (the global figure is $\$ 2.5$ billion). Second, in our world there is nothing which is truly remote and no one from whom we are disconnected. AIDS has again demonstrated that; another example, dengue haemorrhagic fever, which has been ravaging the Caribbean, and for which an effective vaccine does not yet exist, is predicted to spread in epidemic proportions to parts of the United States. Finally, it is becoming more and more clear that poverty and disease have not only a moral impact but a political price. Ultimately, what is the cost of political turmoil? We have the resources to make vaccines and essential drugs available to the people of the Third World; what we need are the imagination and the will.

Barry R. Bloom is in the Department of Microbiology and Immunology, Albert Einstein College of Medicine, 1300 Morris Park Avenue, Bronx, New York 10461, USA.

1. State of the World's Children (UNICEF, Oxford, 1988)

2. World Development Report (The World Bank, Oxford, 1988).

3. World Debt Tables, 1988. (The World Bank, Oxford, 1988)

4. Walsh, J.A. Establishing Health Priorities in the Developing World (United Nations Development Programme, New York, 1988).

5. Henderson, R. in Biomedical Science and the Third World: Under the Volcano (eds Bloom, B.R. \& Cerami, A.) (New York Academy of Sciences, in the press).

6. Moss, B. \& Flexner, C. A. Rev. Immun. 5, 305-324 (1987).

7. Fenner, F. Annis inst. Pasteur (in the press).

8. Morin, J.R. et al. Proc. natn. Acad. Sci. U.S.A. (in the press).

9. Hogle, J.M. Scient. Am. 256, March 42-49 (1987).

10. Burke, K.L., Glynnis, D., Morag, F., Minor, P.D. \& Almond, J.W. Nature 332, 81-82 (1988)

11. Murray, M.G. et al. Proc. natn. Acad. Sci. U.S.A. 85 , 3203-3207 (1988).

12. Nomoto, A., Lizuka, N., Kogara, M. \& Arita, M. Vaccine 6. 134-137 (1988)

13. Rice, C.M. et al. The New Biologist (in the press)

14. Hoiseth, S.K. \& Stocker, B.A.D. Nature 291, 238-9 (1981).

15. Curtiss, R. III \& Kelley, S.M. Infect. Immunity 55, 30353043 (1987).

16. Snapper, S.B. et al. Proc. natn. Acad. Sci. U.S.A. 85 , 6987-6991 (1988)

17. Talwar, G.P. (ed.) Immunological Approaches to Contraception and Promotion of Fertility (Plenum, New York 1986)

18. Schultz, T.W. Investing in People (University of California, Berkeley, 1982).

19. Robbins, A. \& Freeman, P. Scient. Am. 258, November 126-131 (1988).

20. Statistical Abstract of the United States (Government Printing Office. Washington, D.C., 1988). 